Revue des patrimoines

\title{
Vers une ontologie de domaine pour l'analyse de l'état de conservation du bâti patrimonial
}

Towards an ontology for the analysis of the conservation status of a heritage building

Tommy Messaoudi, sous la direction de Livio De Luca, Philippe Véron et Gilles Halin

\section{OpenEdition Journals}

Édition électronique

URL : http://journals.openedition.org/insitu/22470

DOI : $10.4000 /$ insitu. 22470

ISSN : $1630-7305$

Éditeur

Ministère de la Culture

\section{Référence électronique}

Tommy Messaoudi, sous la direction de Livio De Luca, Philippe Véron et Gilles Halin, « Vers une ontologie de domaine pour l'analyse de l'état de conservation du bâti patrimonial », In Situ [En ligne], 39 | 2019, mis en ligne le 13 juin 2019, consulté le 14 juillet 2019. URL : http:// journals.openedition.org/insitu/22470; DOI : 10.4000/insitu.22470

Ce document a été généré automatiquement le 14 juillet 2019

\section{cc) (i)}

In Situ Revues des patrimoines est mis à disposition selon les termes de la licence Creative Commons Attribution - Pas d'Utilisation Commerciale - Pas de Modification 4.0 International. 


\section{Vers une ontologie de domaine pour l'analyse de l'état de conservation du bâti patrimonial}

Towards an ontology for the analysis of the conservation status of a heritage building

Tommy Messaoudi, sous la direction de Livio De Luca, Philippe Véron et Gilles Halin

\section{Introduction, contexte et objectifs}

1 La description, l'analyse et le suivi de la conservation du patrimoine culturel bâti s'opèrent au moyen d'un nombre élevé et très hétérogène de sources documentaires (textes, images, enregistrements vocaux) et de données analytiques (provenant d'analyses d'échantillons, de capteurs divers, des cartographies provenant d'imagerie scientifiques, etc.). D'une part, ces ressources permettent de fournir des informations historiques, archéologiques et constructives nécessaires pour comprendre la structure et l'évolution temporelle de l'ouvrage patrimonial étudié. D'autre part, malgré une certaine complémentarité, ces multiples données sont très souvent disjointes les unes des autres et ne sont pas mises en relation au sein d'un support de représentation commun. De plus, le caractère hétérogène de ces données est la preuve qu'elles ont été produites dans le cadre de pratiques scientifiques et processus métiers divers établis dans le but de créer de nouvelles informations, voire de nouvelles connaissances. Cette diversité d'experts, intervenant dans le projet de restauration du bâtiment, nécessite donc de fédérer et d'archiver de manière cohérente l'ensemble des données produites par chaque corps de métier.

2 Au vu des avancées technologique d'acquisition 2D/3D, le principal problème rencontré par la communauté scientifique du patrimoine culturel a trait à la possibilité de corréler ces données hétérogènes. Cette corrélation permettrait de produire des informations 
pertinentes (reliées à des connaissances consolidées) pour décrire l'état de conservation du bâti. Un autre point important est l'absence de référencement spatial. En effet, même si les données produites se réfèrent à un objet physique commun (un édifice patrimonial par exemple), ou une région spatiale spécifique de cet objet (un mur ou même une forme de dégradation), le lien entre ces données est seulement basé sur une description conceptuelle du bâti (sans aucune référence de leurs positions spatiales réciproques).

3 L'objectif que s'est donné le projet MONUMENTUM ${ }^{1}$ (MOdélisation NUMérique et gEstion de doNnées pour la conservaTion des strUctures Maçonnées), financé par l'ANR est de concevoir un modèle ontologique pour l'observation multidisciplinaire de l'état de conservation, solution semblant aujourd'hui prometteuse pour corréler et structurer les données au sein de représentations 3D sémantiques de bâti patrimonial. Le présent article introduit la conception d'une ontologie de domaine dédiée à l'annotation sémantique 2D/3D de l'état de conservation. Par la combinaison des descripteurs quantitatifs et qualitatifs d'annotations 3D interconnectées, cette ontologie dédiée intègre les données, informations et connaissances qui décrivent et suivent les phénomènes de dégradation afin d'assister les experts du patrimoine culturel au sein d'un processus d'aide à la décision.

\section{État de l'art : revue documentaire}

Chaque acteur de la conservation (ingénieur spécialiste dans l'analyse des matériaux, chercheurs, architecte, restaurateur, conservateur) effectue des diagnostics à travers l'analyse de données hétérogènes. Pour couvrir l'étude complète du domaine de la conservation, l'ensemble des experts passe généralement par l'observation de trois composantes essentielles de l'objet patrimonial: l'apparence visuelle de la surface (imageries scientifiques 2D), la forme géométrique des composants structurels (imageries scientifiques 3D), et enfin la composition et le comportement du matériau de construction par des études physico-chimiques (essais et simulations en laboratoire) (fig. 1). La mise en relation de ces données et leur contextualisation en font des informations. Lorsque celles-ci permettent de créer des savoir-faire sur un phénomène, elles atteignent un niveau d'abstraction sémantique supplémentaire: la connaissance. Cette dernière est la plupart du temps issue de réflexions consensuelles et collaboratives sur un phénomène observé, dont le croisement mène à la production d'ouvrages de référence : des glossaires.

Cette section est présentée en deux parties. La première définit ce que l'on entend par " ontologie de domaine ", afin de mieux comprendre les concepts manipulés dans la prise en compte du suivi de la conservation du patrimoine. La seconde partie rassemble les travaux suggérant des solutions pour pallier aussi efficacement que possible les problématiques énoncées précédemment. Des travaux dont les avantages ont été exploités en tant que point de départ pour notre approche méthodologique. 
Figure 1

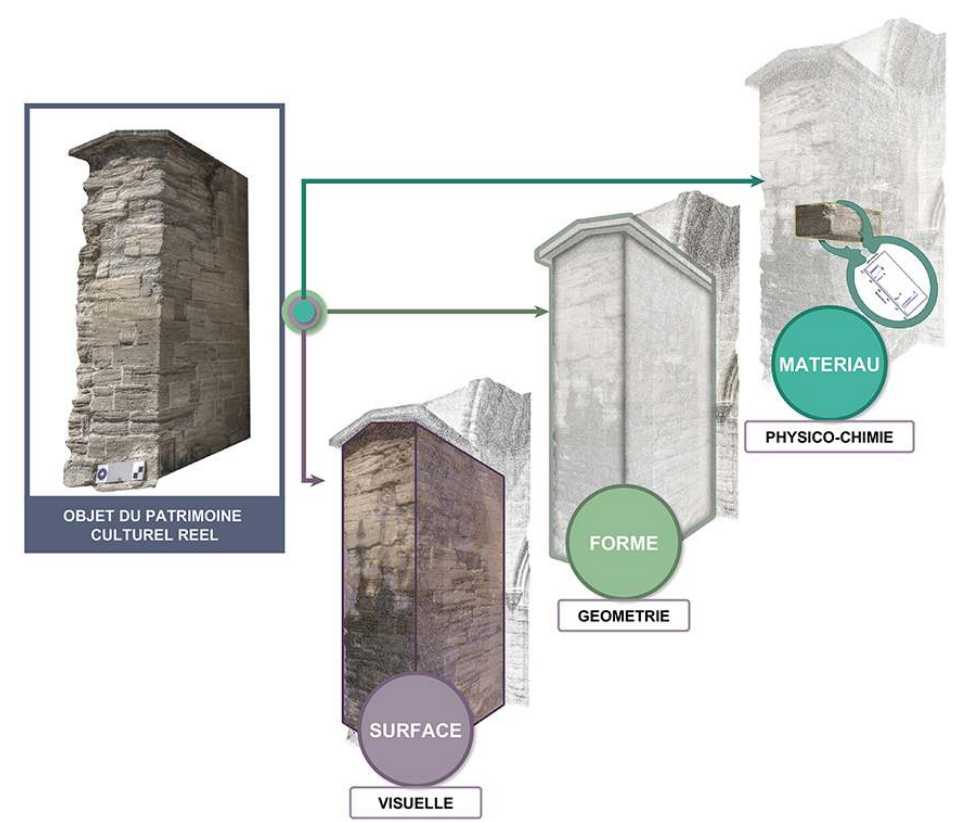

Trois composantes d'études pour l'étude de la conservation du patrimoine culturel bâti.

(c) Tommy Messaoudi, MAP-GAMSAU.

\section{Définition : l'ontologie de domaine}

Une ontologie est utilisée pour décrire, partager, et réutiliser les données, informations et connaissances entre les humains et les agents logiciels. Ce modèle conceptuel est utilisé dans de nombreux systèmes d'information en s'appuyant sur des technologies du web sémantique $^{2}$. Dans le domaine des sciences informatiques et de l'ingénierie des connaissances en particulier, l'ontologie générique ou de domaine est caractérisée comme "une spécification explicite et formelle d'une conceptualisation partagée " ${ }^{3}$. Le terme « spécification explicite » fait référence à la présence de concepts, de relations, de contraintes, de règles, de fonctions et d'axiomes du domaine dans lequel on se trouve, permettant de le décrire le plus précisément et le plus concrètement possible. En outre, cela signifie également que les types de concepts utilisés et les contraintes sur leur utilisation sont explicitement définis. Cette spécification peut être représentée dans un langage formel ou naturel. Le terme « formel » signifie que l'ontologie est une entité qui doit être compréhensible par l'ordinateur. Cette dernière notion est fortement liée à la «conceptualisation». Cette composante concerne la modélisation abstraite et conceptuelle, que l'on veut définir à travers des phénomènes intervenant dans un domaine spécifique. En outre, cette modélisation passe par la structuration de termes et de concepts. Et enfin, "partagée » est l'un des termes principaux qui renvoient au partage et à l'utilisation des connaissances d'un domaine, la plupart du temps composé de plusieurs sous-domaines d'expertise fournissant des connaissances orientées et spécifiques. La modélisation n'est donc pas propre à un individu, mais est acceptée consensuellement par un groupe ou une communauté scientifique intégrant leurs connaissances. 


\section{État de l'art sur les systèmes de gestion dans le domaine du patrimoine culturel bâti}

7 Deux aspects peuvent être mis en évidence dans le domaine de la conservation. Plusieurs systèmes d'information suggèrent de gérer une importante quantité de données, mettant l'accent sur l'aspect quantitatif, tandis que d'autres accordent de l'importance à l'aspect qualitatif mis en évidence à travers une formalisation des connaissances. Cependant, peu d'études intègrent les deux aspects au sein d'une unique approche. Nous présentons ici trois systèmes qui ont tenté d'exploiter cette dualité et nous les accompagnons d'une grille d'évaluation croisant les dimensions relevées et les aspects diagnostiques.

\section{Base de données 3D dédiée à la conservation}

Dans le cadre du projet $\mathrm{SACRE}^{4}$, un système dédié à la documentation numérique pour le suivi des altérations de la pierre a été conçu. La particularité de cet outil est qu'il permet de réaliser des annotations sur des représentations 3D volumiques texturées en utilisant des orthophotographies mappées sur le modèle 3D. Les annotations sont comparées entre elles par des requêtes: comme des analyses statistiques, des calculs de distribution spatiale de cartographie à la volée, et des calculs d'intersection (fig. 2).

Figure 2

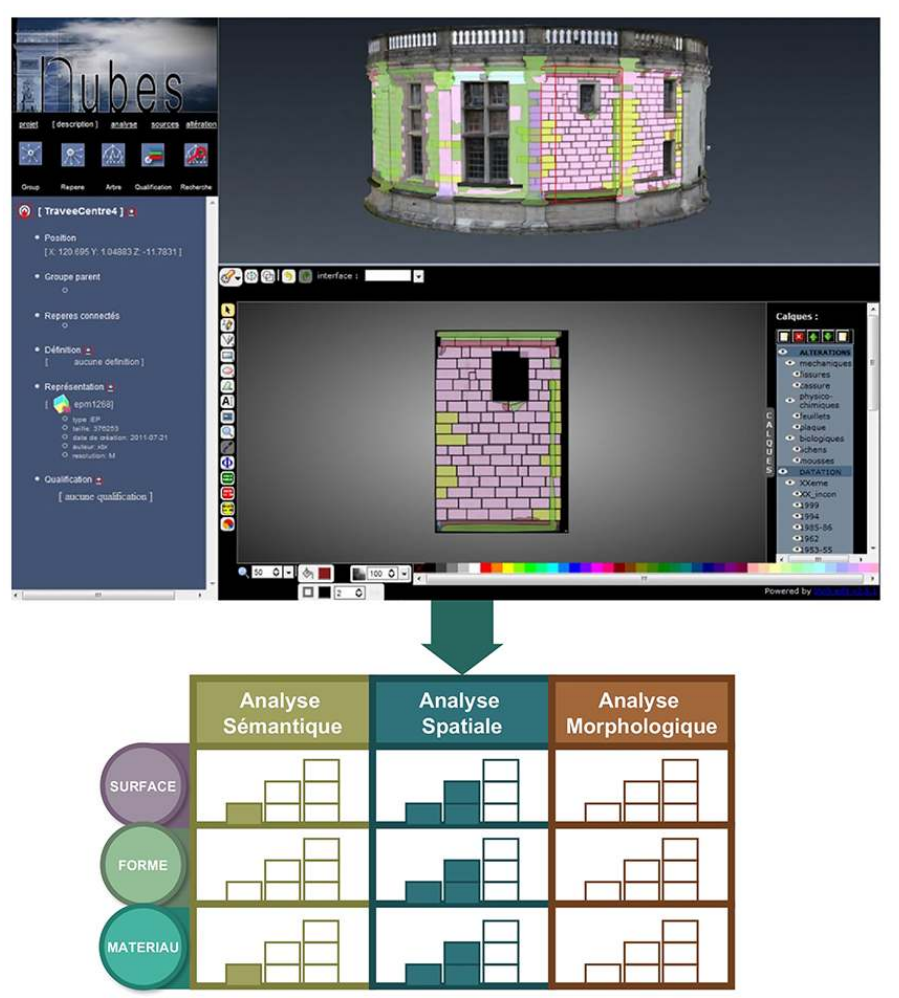

Évaluation de système de gestion de base de données 3D NUBES SACRE.

(c) Tommy Messaoudi, MAP-GAMSAU.

Ce travail est vraiment intéressant et constitue une première tentative en termes de spatialisation d'informations. Cependant, malgré la richesse des données acquises et 
l'utilisation de termes de description, ce système n'est pas suffisant pour décrire et représenter l'ensemble du processus d'observation scientifique des différents aspects diagnostiques. Enfin, il ne propose aucune possibilité d'extraire des informations morphologiques relatives à de tels aspects.

10 Le projet SACRE constitue le résultat qui détermine la nécessité de construire une ontologie. Qu'en est-il des systèmes basés sur les connaissances qui exploitent ce type de méthode?

Le Modèle PARCOURS : PAtrimoine culturel et Restauration-Conservation : Ontologie pour l'Usage d'un Référentiel commun aux différentes Sources de données

11 Le modèle "Parcours $»^{5}$ est un travail en cours qui traite de la documentation du patrimoine à l'aide d'une ontologie de référence standardisée, le CIDOC-CRM (section 3.3.1.). Son objectif est de lever un verrou scientifique relatif à l'harmonisation des données provenant de sources distinctes. Il se base entre autres sur les bases de données CASTOR et IMAGE. Ces dernières renferment des ressources documentaires et analytiques instrumentales. La figure 3 est un exemple de diagnostic concret permettant de comprendre les potentialités de ce modèle (fig. 3). Le cas d'étude concerne notamment la restauration d'un vitrail de la Sainte-Chapelle (Paris), conduite par l'équipe du LRMH (Laboratoire de recherche des monuments historiques) : «La baie 109 du Mur Nord de la Sainte-Chapelle a fait l'objet d'un diagnostic réalisé par Fanny Bauchaut. Elle a identifié une altération de type Dépôt situé sur le bord du vitrail de la Fenêtre a28. Une analyse d'échantillon a été réalisée et a détecté un agent de dégradation: le gypse. Le dépôt a ensuite été nettoyé avec du bicarbonate d'ammonium ». 


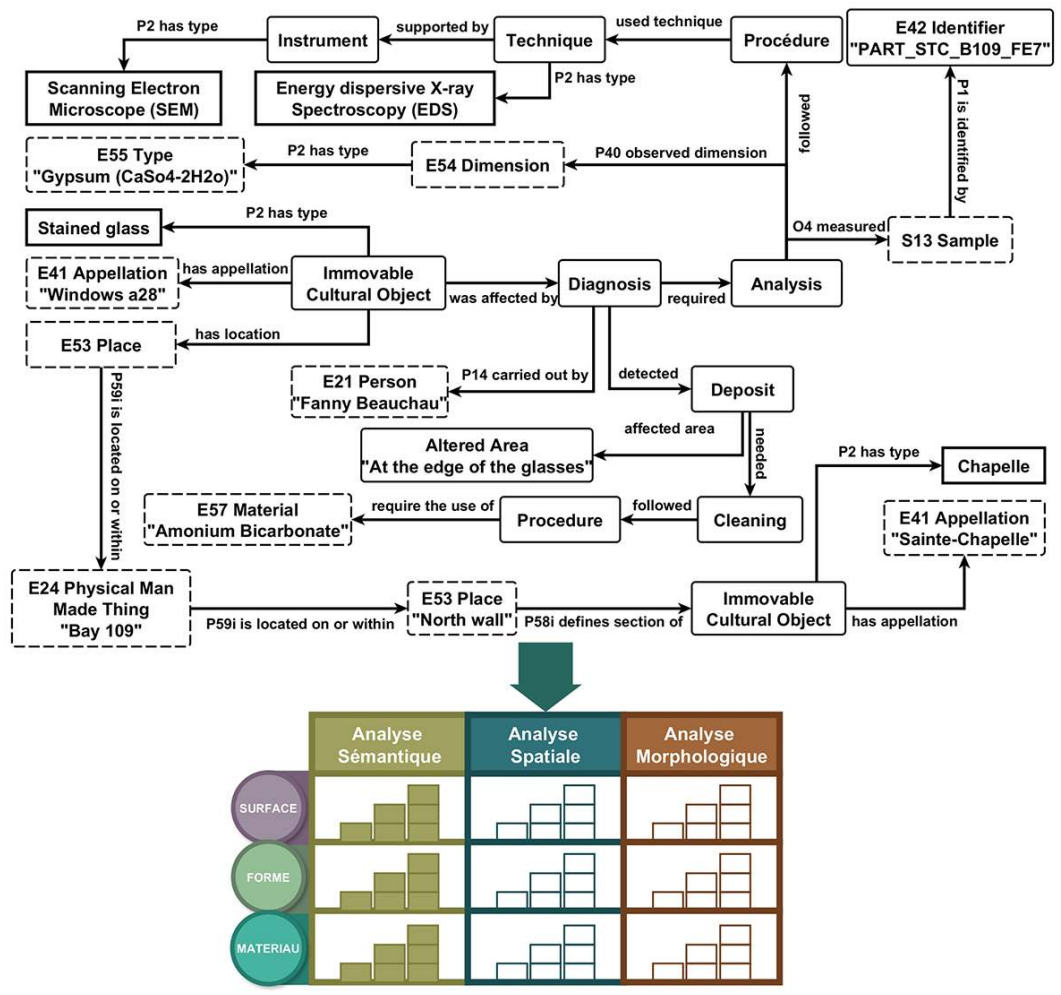

Cas d'étude applicable sur une portion du modèle PARCOURS et évaluation.

(c) Tommy Messaoudi, MAP-GAMSAU et Cheikh Niang, LRMH.

L'utilisation d'une ontologie de référence mappée sur une ontologie dédiée permet la description des observations, des procédures scientifiques et des données impliquées. Cependant, la description spatiale est seulement basée sur une suite d'identifiants, ce qui ne permet pas de constater visuellement ni morphologiquement les observations diagnostiques sur un support d'analyse.

\section{Une ontologie dédiée aux altération du patrimoine : MONDIS}

Le troisième exemple, MONDIS (MONument Damage Information System) ${ }^{6}$, est un système dédié à la gestion des phénomènes d'altération. Il a la particularité d'être basé sur un traitement automatique croisant plusieurs aspects : la documentation sur les altérations du patrimoine, leur diagnostic et enfin les interventions de restauration. Le modèle proposé peut être simplement décrit comme suit: un événement, accompagné d'une référence temporelle définie, induit un mécanisme qui peut être réalisé par un agent. Le mécanisme de dégradation produit une altération tangible et observable sur le bâti, appelé manifestation de la dégradation, et inclut deux entités : le matériau et le type de composant d'altération. La phase d'intervention est modélisée comme une action (ou ensemble d'actions). Elle est ciblée pour remédier à cette manifestation, stoppant le mécanisme de dégradation en éliminant l'agent de dégradation identifié afin d'empêcher que l'événement se renouvelle. Voici un exemple concret de son utilisation (fig. 4): "Basé sur les images acquises in situ, une fissure verticale a été détectée. Elle s'est formée suite à la construction d'un toit datant de 1970 qui agit en compression sur le pilier. D'après une acquisition in situ cette ouverture mesure $1 \mathrm{~mm}$ d'épaisseur. Grâce à 
l'utilisation d'un modèle de calcul, la charge statique qui est responsable de cette compression est de 1,6 hPa (hectopascal). Elle est stoppée grâce à une technique de confinement $»$.

Figure 4

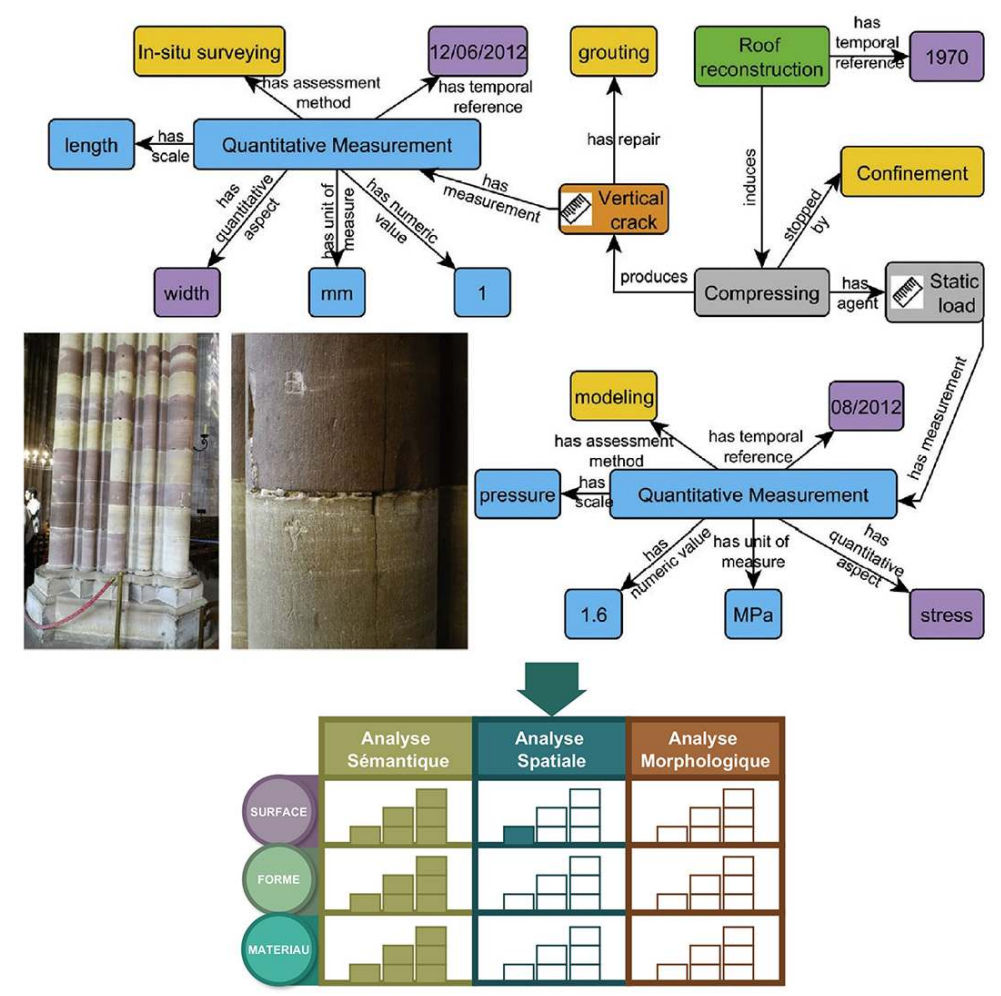

Cas d'étude réel insérable dans MONDIS sous forme de modèle conceptuel et évaluation dimensionnelle.

(c) Tommy Messaoudi, MAP-GAMSAU et Riccardo Cacciotti, ITAM.

14 La description des observations de terrain et en laboratoire est un point fort de ce système. Cependant, même s'il fait usage de photographies pour documenter le diagnostic, MONDIS n'utilise en aucun cas des annotations spatialisées permettant de guider l'utilisateur dans sa recherche d'information. Enfin, il n'y a pas d'information relative à l'analyse morphologique qui mette en exergue les conséquences des contraintes environnementales.

\section{Bilan et constats sur l'état de l'art}

Après l'analyse de ces outils, on relève deux orientations en rapport avec une entité observable du bâtiment : une quantitative, l'autre qualitative. Le projet SACRE propose une solution de spatialisation très efficace en matière d'analyse quantitative de la distribution spatiale. Mais malgré l'utilisation de termes décrivant les artefacts patrimoniaux, elle ne renferme pas un modèle de connaissances intégrées préétabli pour décrire formellement les phénomènes d'altération. À l'inverse, MONDIS et PARCOURS mettent davantage en évidence les aspects qualitatifs car ils exploitent un modèle de connaissances riche. C'est pourquoi nous voulons, à travers notre approche, tirer parti de leurs avantages: ceux du système NUBES SACRE qui rendent possible le référencement spatial, puis ceux du système MONDIS et PARCOURS qui proposent une documentation 
cohérente en matière d'observation des phénomènes de dégradation. Ainsi, nous pouvons formuler cette hypothèse : un système d'information, structuré par une ontologie de domaine multidimensionnelle, permet d'exploiter tout le spectre quantitatif/qualitatif dans l'analyse des aspects diagnostiques de la conservation du patrimoine.

\section{Une ontologie de domaine dédiée à la spatialisation des observations scientifiques menées sur le bâtiment}

\section{Orientation générale de l'approche méthodologique}

16 L'hypothèse précédemment citée peut être représentée sous la forme de la figure 5 (fig. 5). Afin d'étudier l'état de conservation d'un objet physique patrimonial (1.), les experts de la conservation (2.) mènent une panoplie d'actions (3.) produisant données et informations.

Figure 5

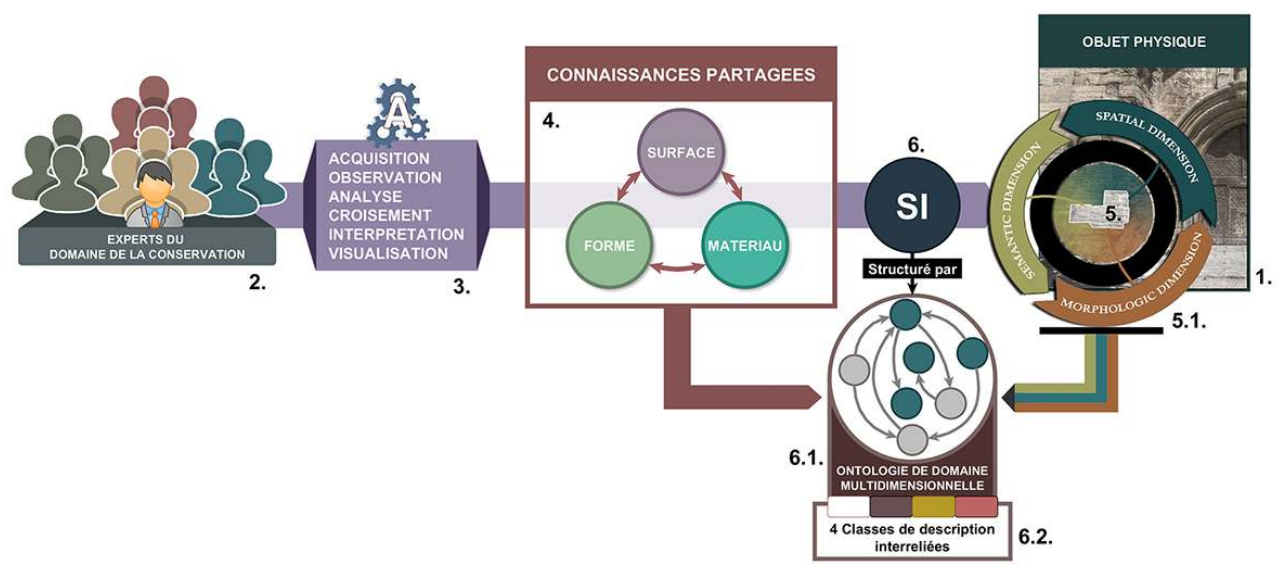

Proposition d'une nouvelle approche pour l'étude de la conservation du patrimoine architectural. (c) Tommy Messaoudi, MAP-GAMSAU.

Pour cela, ils s'appuient sur les connaissances partagées relatives aux aspects diagnostiques identifiés (4.). Nous savons également que pour une observation donnée, l'objet peut être décrit sous trois dimensions (5.1.): sémantique, spatiale et morphologique. Elles n'ont pour le moment jamais fait l'objet d'une interconnexion fonctionnelle. L'aspect innovant de cette contribution scientifique est que, pour la première fois dans le domaine de la conservation, nous concevons un système d'annotation (6.) pour la collecte et l'acheminement d'informations basé sur une ontologie de domaine (6.1.). Une ontologie qui est non seulement dédiée à la description des aspects diagnostiques (4.) mais qui relie également les trois dimensions (5.1.) au sein d'un unique modèle de connaissances. Le levier essentiel de cette interconnexion repose sur la définition de quatre classes de descriptions nécessaires et suffisantes (6.2.). Elles constituent les fondations de notre échafaudage conceptuel pour clarifier l'ensemble des interrelations qui permettent, par combinaison, de décrire les phénomènes de dégradation du bâti. 


\section{Activité du moteur de corrélation multidimensionnel}

La figure suivante présente la description des activités systèmes sur laquelle nous nous sommes basés pour développer notre approche (fig. 6). Pour l'étude d'un objet physique patrimonial, quatre activités se déroulent successivement :

A1, l'expert a la possibilité de spatialiser des données acquises in situ telles que des images dans un système de référence tridimensionnel.

A2, le même expert ou même un second, peut réaliser des annotations sur le support d'analyse 2D/3D basé sur les images, représenté ici par la zone blanche, parce qu'il a reconnu et identifié quelque chose.

A3, à la volée, l'annotation 2D/3D est ensuite soumise à une analyse morphologique.

Enfin, A4 est dédiée à l'étape de désignation. L'expert assigne un concept parmi les quatre classes de descriptions essentielles de notre ontologie : Altération, Matériau, Technique de construction, et Composants architecturaux. L'expert argumente son choix en s'appuyant sur un document de la classe acquisition. Les trois dimensions sont donc exploitées dans le système et permettent de fédérer l'ensemble des données et de guider l'expert dans son observation au sein d'une représentation 3D.

Figure 6
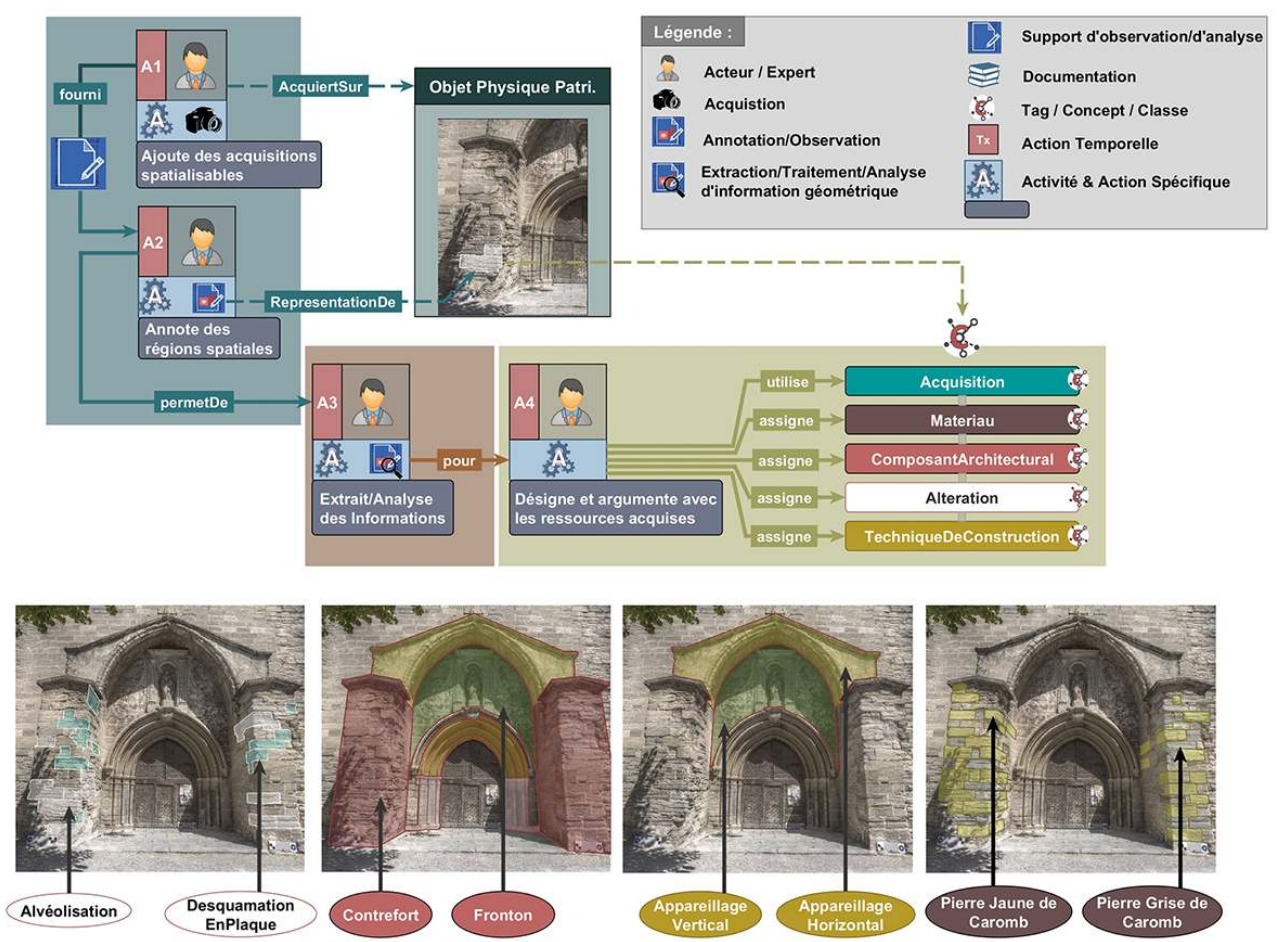

Approche générale du système de corrélation avec exemples.

(c) Tommy Messaoudi, MAP-GAMSAU.

\section{Une ontologie de domaine dédiée à la conservation}

Notre ontologie de domaine est la clé de voûte qui permet de mettre en relation les connaissances partagées et les trois dimensions. Elle comprend quatre classes nécessaires 
et suffisantes de descriptions thématiques reliées entre elles par des relations spécifiques appelées propriétés d'objet. La Figure 7 décrit la chaîne conceptuelle résultante : « un matériau est utilisé pour une technique de construction spécifique, qui est elle-même utilisée pour former un composant architectural (fig. 7). Puis, une altération agit sur un matériau ». Les propriétés inverses rendent possible le croisement de toutes les données dimensionnelles relatives à chaque classe.

Figure 7

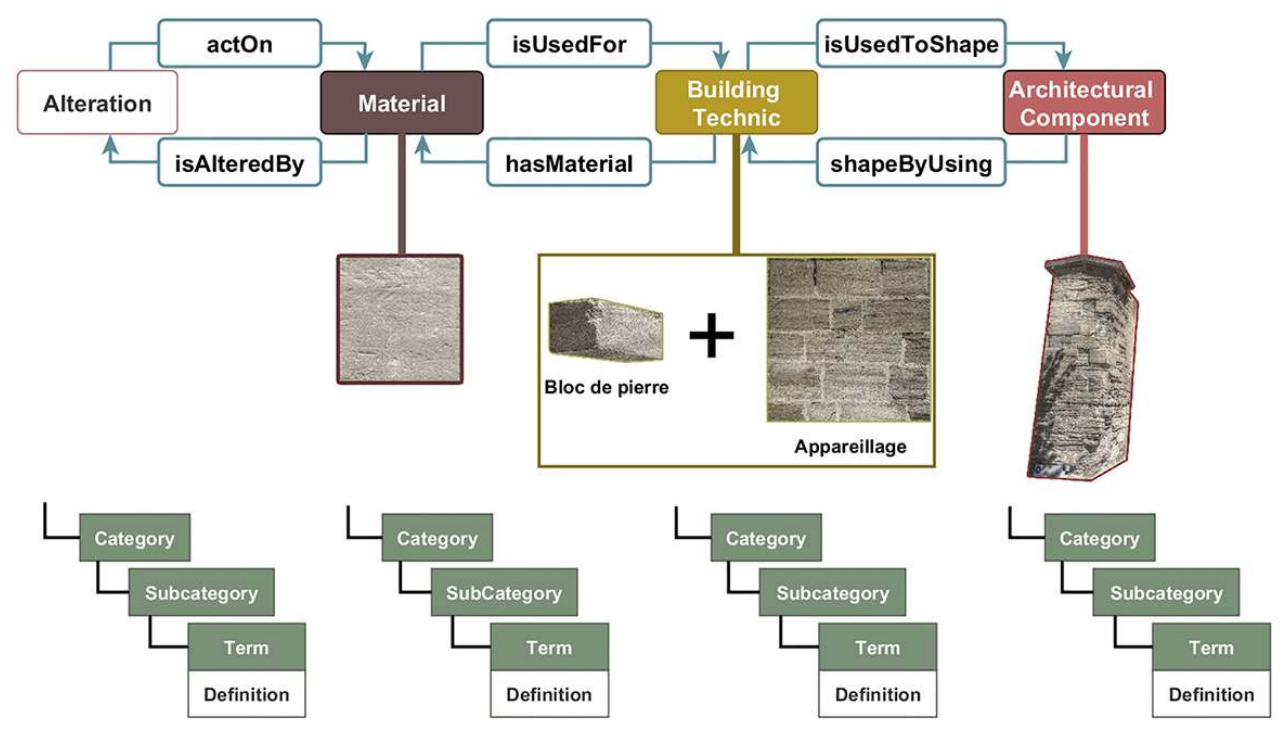

Cœur de notre ontologie dédiée à la conservation.

(c) Tommy Messaoudi, MAP-GAMSAU.

description des données et activités scientifiques. En outre, nous avons utilisé le modèle conceptuel de référence CIDOC-CRM ${ }^{8}$. Cette ontologie fournit une structure formelle pour décrire les concepts explicites et implicites, puis les relations utilisées dans la documentation du patrimoine culturel. Cette modélisation a été initiée lorsque l'on s'est rendu compte qu'il était impossible de créer une base de données unifiée qui documente 
la complexité du patrimoine culturel. Ce modèle générique, nommé CRMCore, propose plusieurs modèles d'extensions exploitables. CRMsci pour décrire les pratiques d'observation scientifique, CRMdig pour décrire les pratiques de production de données numériques, et enfin, CRMinf pour renforcer l'argumentaire d'une observation scientifique.

La fusion du CIDOC-CRM et de notre ontologie permet d'obtenir un modèle sémantique complet pour les objets du patrimoine et les activités scientifiques (fig. 8).

Figure 8

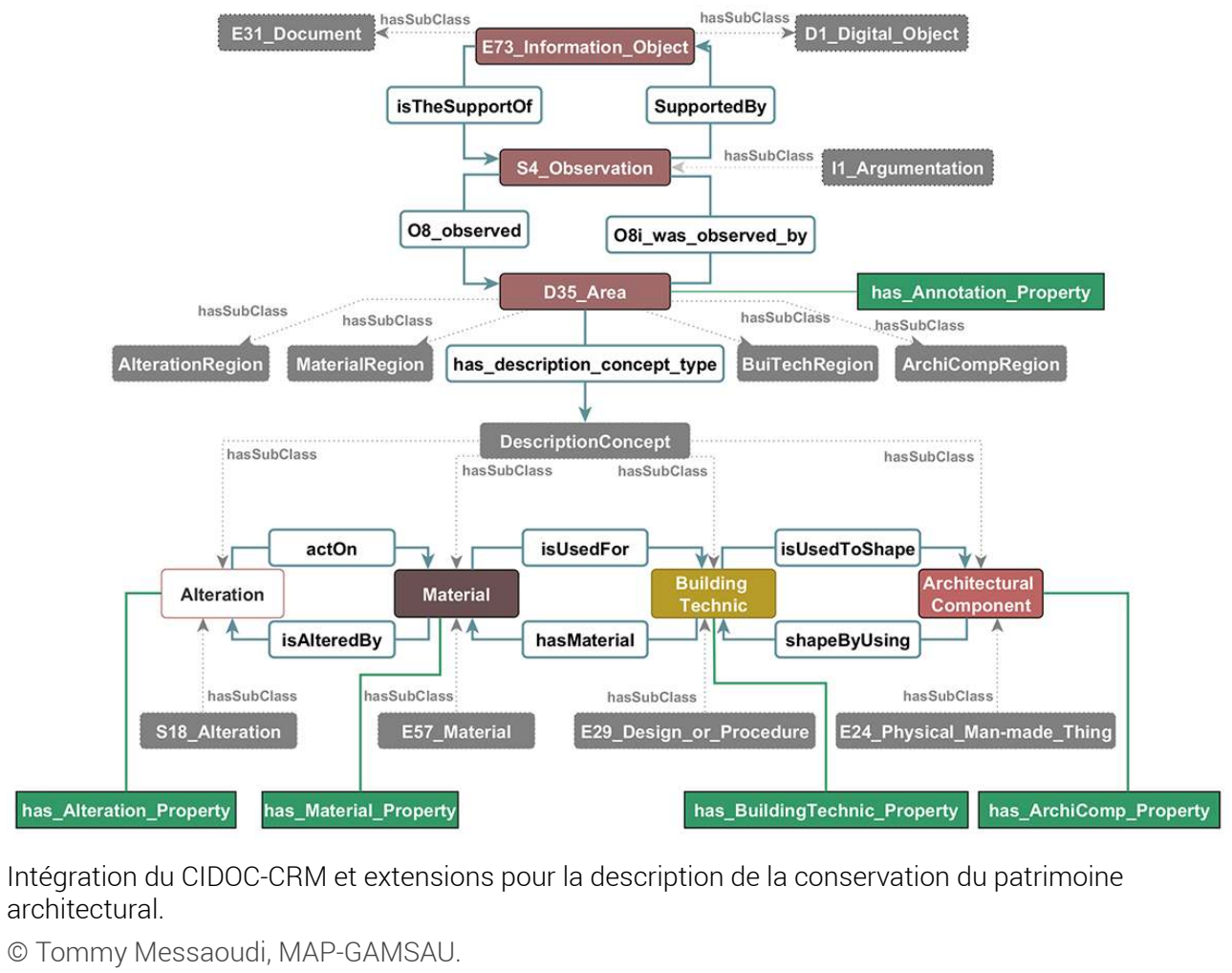

L'implémentation de notre ontologie s'est orientée vers l'application d'un outil basé sur l'annotation sémantique d'images 2D/3D. Une observation (S4_Observation) implique donc d'identifier une région (D35_Area) et de lui assigner une connaissance parmi les quatre classes de description. L'observation réalisée recommande également de justifier le terme choisi par l'utilisation d'une référence décrite dans la classe E73_Information_Object, qui regroupe toutes les acquisitions directes et indirectes concernant le bâti étudié (données 3D, données 2D, rapports d'analyses, autres observations, etc.).

29 La dimension spatiale que nous allons présenter dans la partie suivante nous permet de spatialiser ces observations au sein d'un support d'analyse référencé, et d'extraire d'autres descripteurs essentiels.

\section{La dimension spatiale}

La dimension spatiale repose sur la réalisation d'une représentation 3D dense dédiée à la spatialisation d'observations scientifiques. Cette représentation est générée grâce à l'acquisition d'images in situ et à leur corrélation par des méthodes de reconstruction 3D automatiques (fig. 9). Cette dernière crée une relation projective 2D/3D. Les spécificités 
du relevé patrimonial supposent d'acquérir de grandes quantités d'images de tous types pour l'analyse et la documentation de l'objet. Elles contiennent un haut niveau d'information, et constituent donc un support efficace pour effectuer des analyses, qu'elles soient automatiques ou cartographiques.

Figure 9

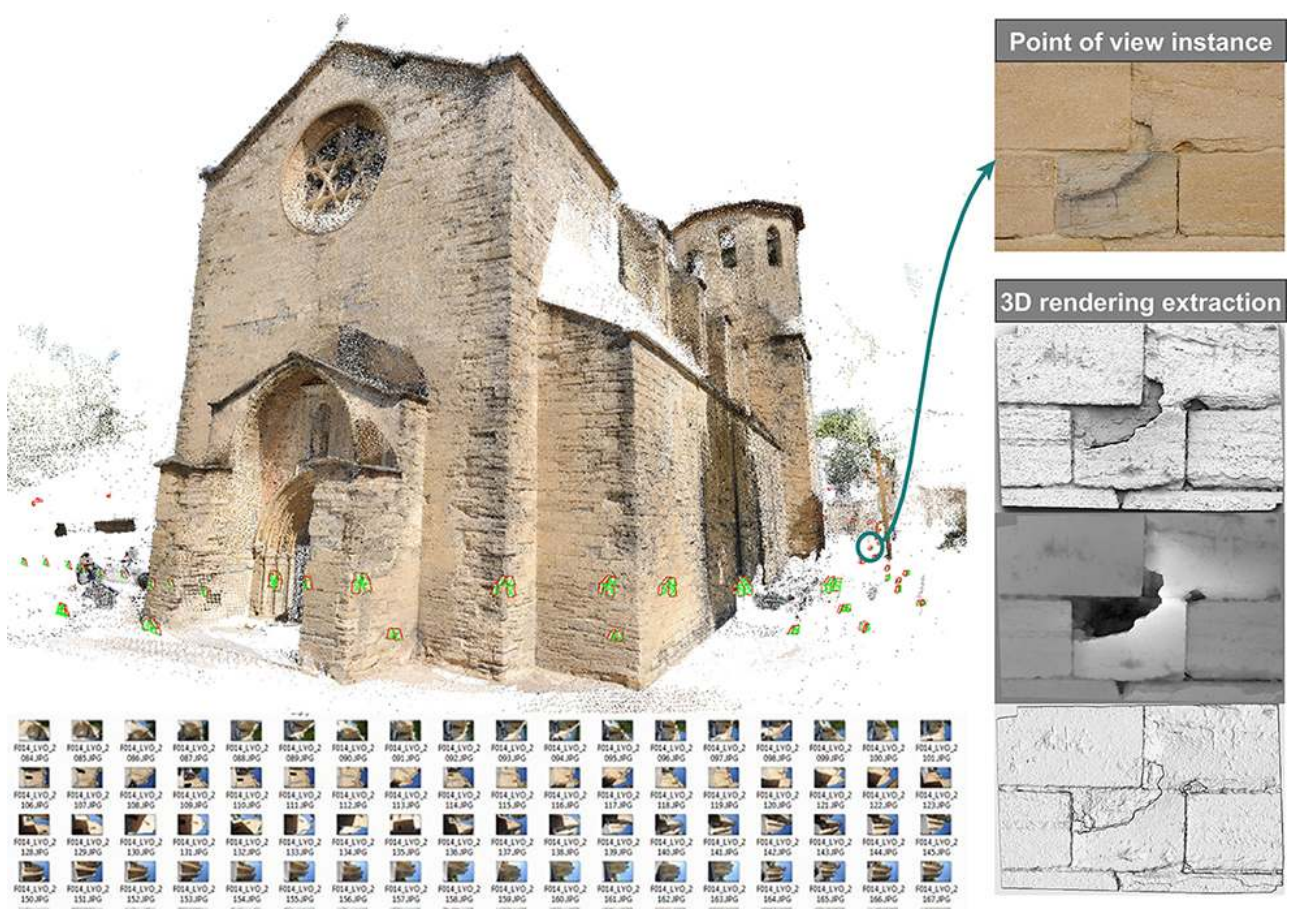

Modélisation 3D basée sur les images, avec des exemples de rendus générés : église Saint-Maurice de Caromb (Vaucluse).

(c) Tommy Messaoudi, MAP-GAMSAU.

31 Ainsi, après la génération de ce nuage de points dense et à partir de la définition d'une annotation sur une image, la relation 2D vers 3D, qui est une phase de transfert, permet d'extraire un nuage de points représentant l'annotation. Puis, la relation 3D vers 2D, qui est la phase de propagation, permet de retrouver automatiquement cette même zone sur les autres images où elle apparait. L'expert peut donc propager des cartographies qu'il réalise à partir d'une seule image.

L'annotation d'image permet d'obtenir un autre niveau d'information pour les points 3D (qu'elle génère sous forme d'index) et pour les annotations: les coordonnées de la position, l'orientation principale, la couleur, ainsi que les valeurs de sa boîte englobante. Cette propagation peut également se faire entre des images de différents types, référencées dans la même base spatiale, comme les images de détails et multi-spectrales.

Cette dimension permettra à court terme d'orienter les images de deux campagnes photographiques d'états temporels T0 et T1. En outre, une annotation de type altération peut être réalisée sur une image de T0, et une autre annotation du même type sur une image de T1 (fig. 10). Dans le cas où les deux acquisitions sont dans le même repère absolu et que les deux régions renvoient spatialement et sémantiquement à la même forme d'altération, l'annotation T0 pourra alors être projetée sur l'annotation T1. Nous pourrons alors quantifier les modifications spatio-temporelles (extension spatiale, 
déplacement du centre de gravité, etc.) de l'état de conservation de l'édifice. L'ontologie actuelle est prévue pour prendre en compte ces aspects.

Figure 10
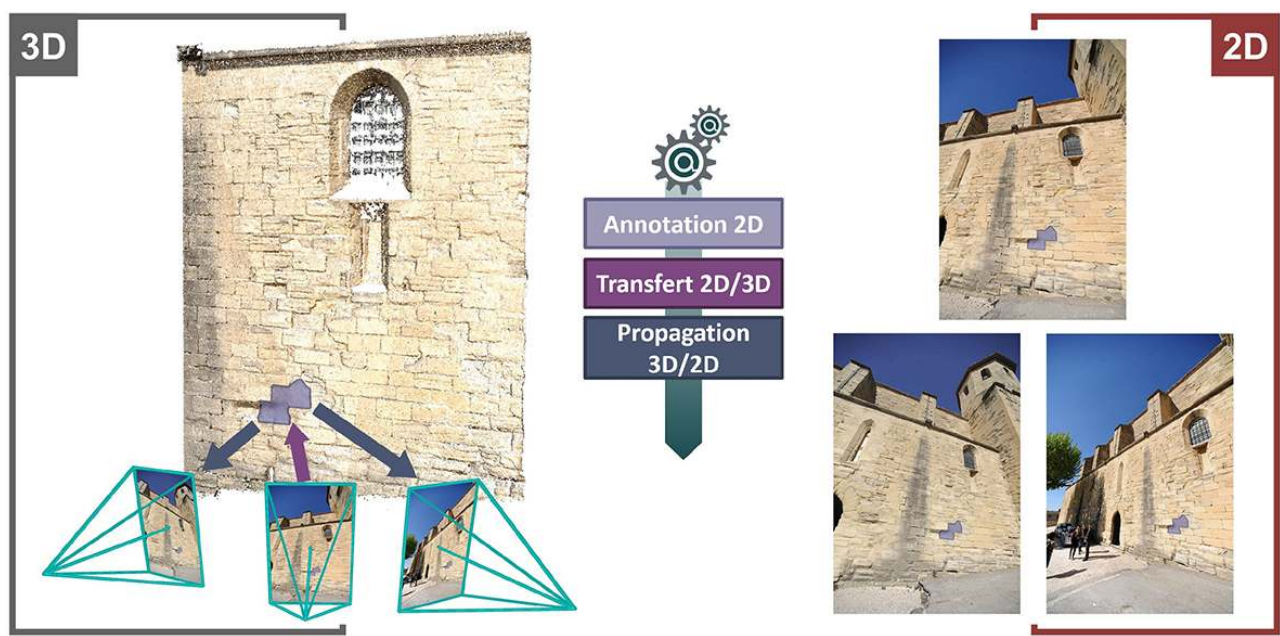

Méthode de transfert d'annotations en utilisant les relations 2D/3D : visualisation 3D à gauche et $2 \mathrm{D}$ à droite.

(c) Tommy Messaoudi, MAP-GAMSAU.

\section{La dimension morphologique}

Ce processus d'annotation peut ensuite être combiné avec une analyse de nuage de points donnant des informations géométrico-visuelles. Deux travaux ont été réalisés en parallèle sur l'église Saint-Maurice de Caromb (Vaucluse) (fig. 11).

Figure 11
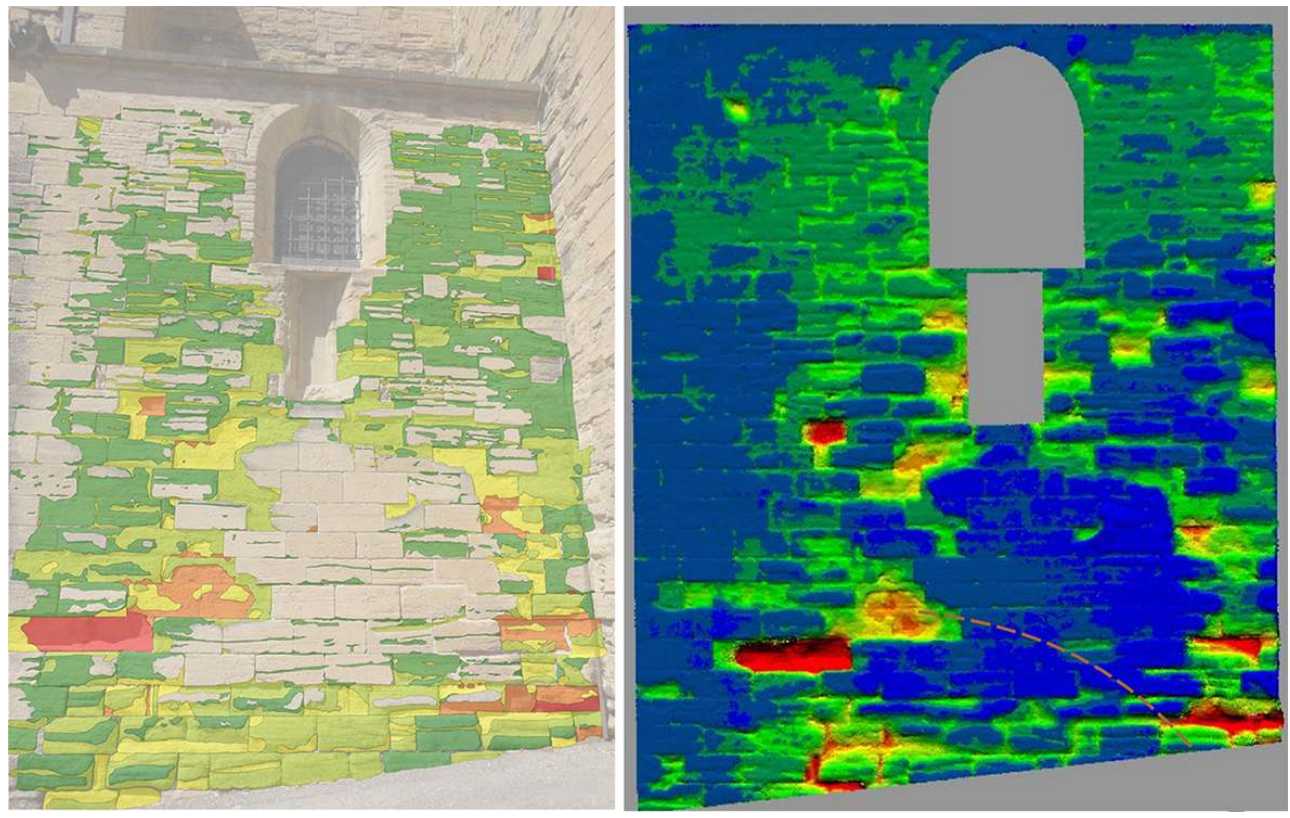

Annotations traditionnelles (à gauche) et analyse géométrico-visuelle automatique du nuage de points (à droite).

(c) Romina Nespeca, MAP et Amélie Benard, CICRP. 
Premièrement, un travail s'est appuyé sur une analyse documentaire et de terrain pour décrire les matériaux de construction, les composants architecturaux et les altérations ${ }^{9}$. Ces restitutions cartographiques manuelles sont considérées comme étant des observations scientifiques. Deuxièmement, un travail a été orienté sur l'extraction de descripteurs géométrico-visuels basés sur les nuages de points reconstruits photogrammétriquement ${ }^{10}$. Par exemple, une analyse RANSAC est un traitement qui permet de visualiser la distribution des sauts de profondeurs à une échelle donnée. La comparaison de ces deux types de cartes montre une certaine robustesse et une fiabilité de la méthode automatique pour le moteur de corrélation.

Grâce à cette dimension, d'autres descripteurs géométrico-visuels peuvent donc être générés : la couleur (RGB), la position (XYZ), les normales (NxNyNz) (qui sont déjà des données produites par la dimension spatiale), les valeurs de profondeur, les valeurs d'occlusion ambiante, etc. Donc, pour un unique point 3D, cette quantité d'informations est disponible. Comme nous l'avons exposé dans la dimension sémantique, les quatre classes de description en interrelation à travers des propriétés objets peuvent être utilisées pour désigner une annotation dans le cadre d'une observation scientifique. Chacune des instances de type région est décrite à travers des données qui la caractérisent. En ce sens, nous exploitons cette dimension pour associer ces annotations à ces descripteurs géométrico-visuels. Leur exploitation en masse devrait faire l'objet d'expérimentations sur des cas réels.

\section{Moteur de corrélation multidimensionnelle}

37 La fusion des trois dimensions nous permet donc de décrire chacune des activités du moteur de corrélation. Sur les différentes figures, le modèle en violet est l'instanciation $\mathrm{du}$ modèle ontologique en rouge. Lors du processus photogrammétrique initié par un expert pour fournir un support 3D dense et cohérent, une image 2D «F014_LYO457 » acquise avec un appareil photographique par « Anthony Pamart » à une date précise, est transformée en image orientée 3D « 457 » à travers un processus d'orientation appliqué par « Frida Peteler » (fig. 12). 
Figure 12

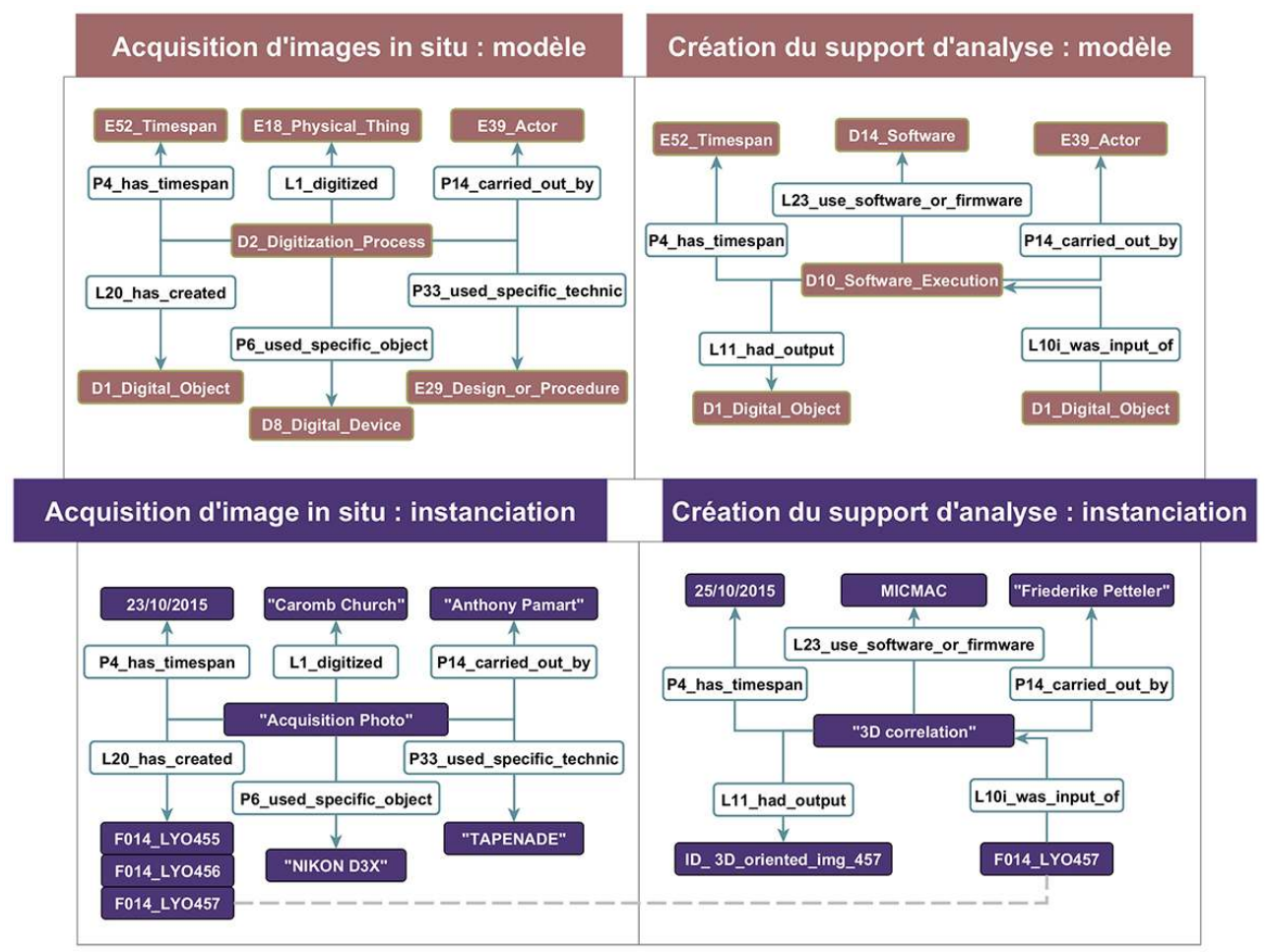

Exemple de description d'une acquisition par le CRMdig pour la provenance des données.

(c) Tommy Messaoudi, MAP-GAMSAU.

Le processus d'observation peut donc être initié (fig. 13). "Sébastien Aze » réalise une observation en identifiant cette annotation « RegionA1 ». Il lui assigne un concept de type « Desquamation en plaque », argumente son choix par un document «ID_Doc_35 » décrit dans la base de connaissances. Plusieurs experts peuvent valider cette observation, voire l'enrichir avec d'autres argumentations. Comme le fait «Jimmy Didouche» lors d'une seconde observation sur la même région. Cette annotation fait ensuite l'objet, à la volée, d'une analyse morphologique qui permet de la décrire spatialement pour l'annotation et géométrico-visuellement pour les index qu'elle englobe.

La fusion de ces dimensions permet de disposer d'un moteur de corrélation multidimensionnel avec des processus dédiés (fig. 14). Basé sur une reconstruction 3D photogrammétrique, une image 2D (1.A.) possède sa représentation 3D (1.B.), orientée autour du nuage de points dense. Le système permet d'annoter sur quatre calques de description thématique via la dimension spatiale, à l'image des classes, notre dimension sémantique qui contient les connaissances partagées. De nombreuses annotations peuvent être faites, et être visibles sur toutes les images grâce à la relation projective 2D/3D. 
Figure 13

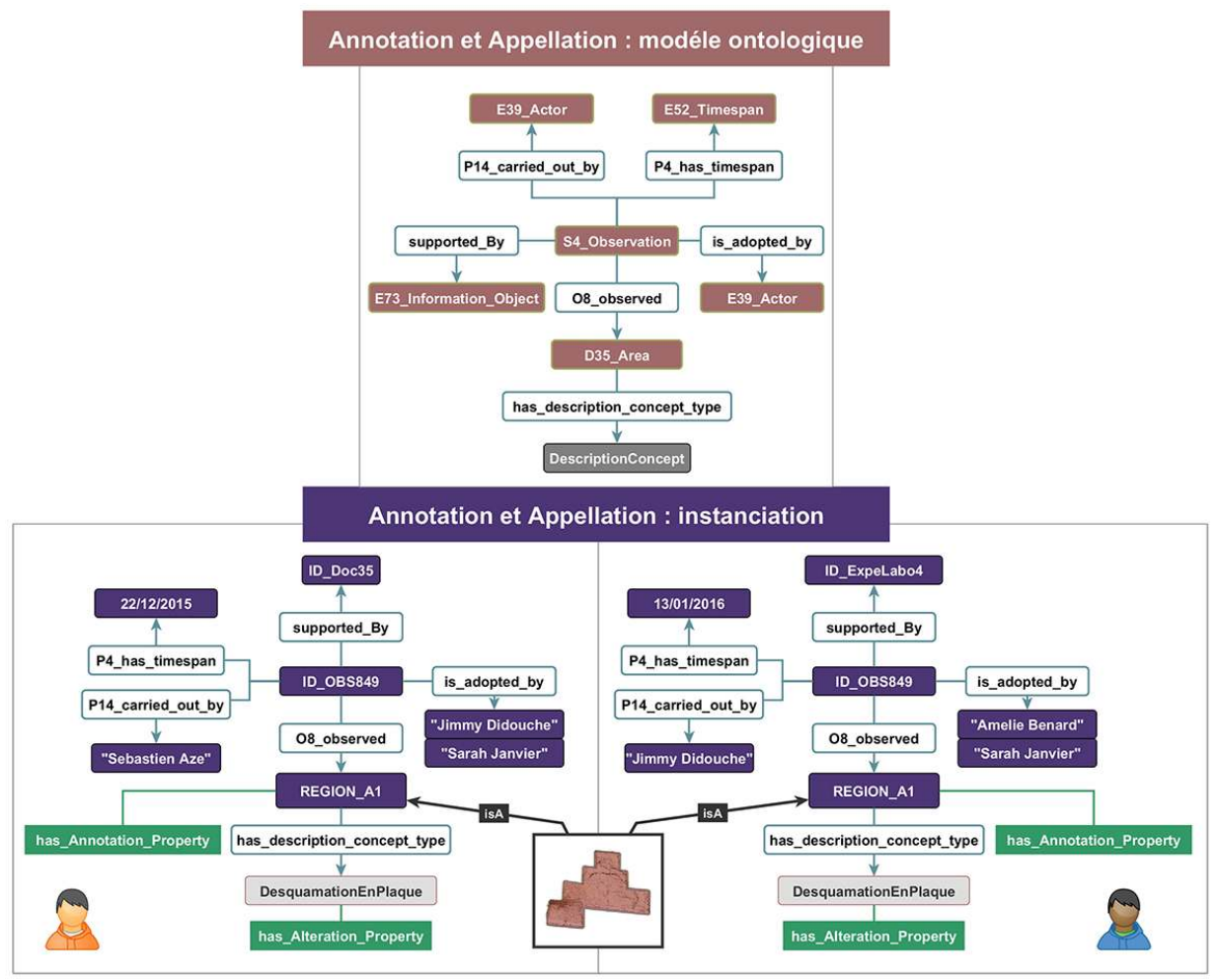

Observations scientifiques d'une altération.

(c) Tommy Messaoudi, MAP-GAMSAU. 


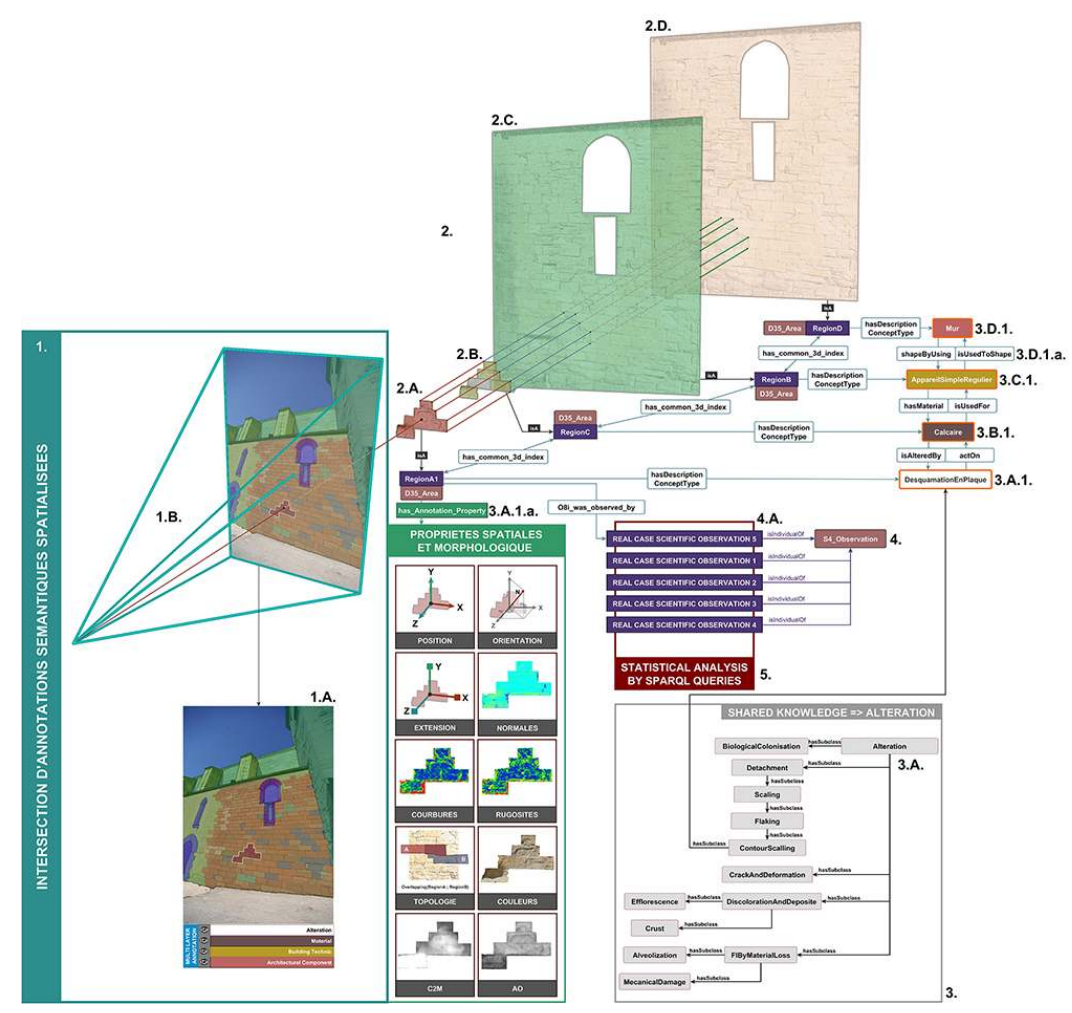

Moteur de corrélation multidimensionnelle.

(C) Tommy Messaoudi, MAP-GAMSAU.

Nous pouvons prendre en considération l'annotation rouge qui se trouve sur le calque altération, et nous projetons les calques 3D (2.) dans l'espace en conservant seulement celles qui se superposent à elle. Avec le modèle de référence CIDOC-CRM, nous pouvons faire des observations (4.). Un expert a donc identifié et dessiné des annotations. Elles portent les identifiants RegionA1, RegionC, RegionB et RegionD.

Lors d'une observation, l'annotation est désignée en choisissant un concept provenant des connaissances partagées et préétablies de la dimension sémantique (3.): donc, pour l' Observation 5 la RegionA1 est reliée à "DesquamationEnPlaque» (3.A.1.), la RegionC à " Calcaire " (3.B.1.), la RegionB à "AppareilSimpleRegulier» (3.C.1.) et la RegionD à «Mur» (3.D.1.). Ces annotations possèdent des index 2D/3D en commun, ils se superposent donc spatialement, mais également sémantiquement à travers les relations préétablies des classes de l'ontologie (3.D.1.a. par exemple). En outre, nous pouvons énoncer :

«RegionA1 (classe Altération: sous-classe Desquamation en Plaque) agit sur RegionC (classe Matériau: sous-classe Calcaire) qui est utilisée pour RegionB (Classe Technique de Construction: sous-classe Appareillage Simple) qui est utilisée pour former RegionD (classe Composant Architectural : sous-classe Mur)».

Chaque région est ensuite décrite par des descripteurs géométrico-visuels issus d'une analyse morphologique (3.A.1.a.). Ce modèle, enrichi par ces instances, peut être utilisé dans le cadre d'autres cas d'étude pour multiplier les individus. Donc, pour un même édifice ou pour d'autres, plusieurs cas réels d'observation scientifique nommés " 1 ", « 2 », « 3 » et « 4 » peuvent identifier des régions spatiales reliées au concept «Desquamation en plaque». Toutes les régions spatiales typées par ce concept seront 
mises en relation, ce qui permettra de réaliser des analyses statistiques de similarité à travers des requêtes SPARQL ${ }^{11}$. L'enjeu à terme est de réaliser des études statistiques sur ces données. Par exemple, pour n'afficher que les dégradations d'un certain type, qui se situent généralement dans les parties basses de l'édifice, il y aura une mise en jeu du barycentre commun, ou encore, nous pourrons constater automatiquement qu'une altération est la plupart du temps superposée à un matériau caractéristique.

\section{Implémentation informatique du système basé sur l'ontologie de domaine}

Pour ce qui concerne l'implémentation, une plateforme web nommée AIOLI ${ }^{12}$, en cours de développement, permet déjà de prendre en charge l'intégration et la corrélation d'images pour reconstruire un nuage de points 3D (fig. 15). Une base de données MySQL dédiée est déjà disponible pour intégrer les données produites. Nous utilisons donc un outil d'accès aux données basé sur l'ontologie, ONTOP, qui permet de mapper notre modèle sur cette base de données. OWL API, utilisé par Protégé, s'occupe de gérer l'ontologie. Le stockage des triplets (données manipulées par une ontologie), sera assuré par la base de données graphe Ontotext. Les experts pourront à tout moment visualiser l'ontologie à l'aide de VOWL, et certains pourront l'éditer grâce à un éditeur web de thésaurus nommé TEMATRES dans un cadre collaboratif. Tematres fournira à l'utilisateur les thésaurus de l'ontologie pour la désignation.

Figure 15

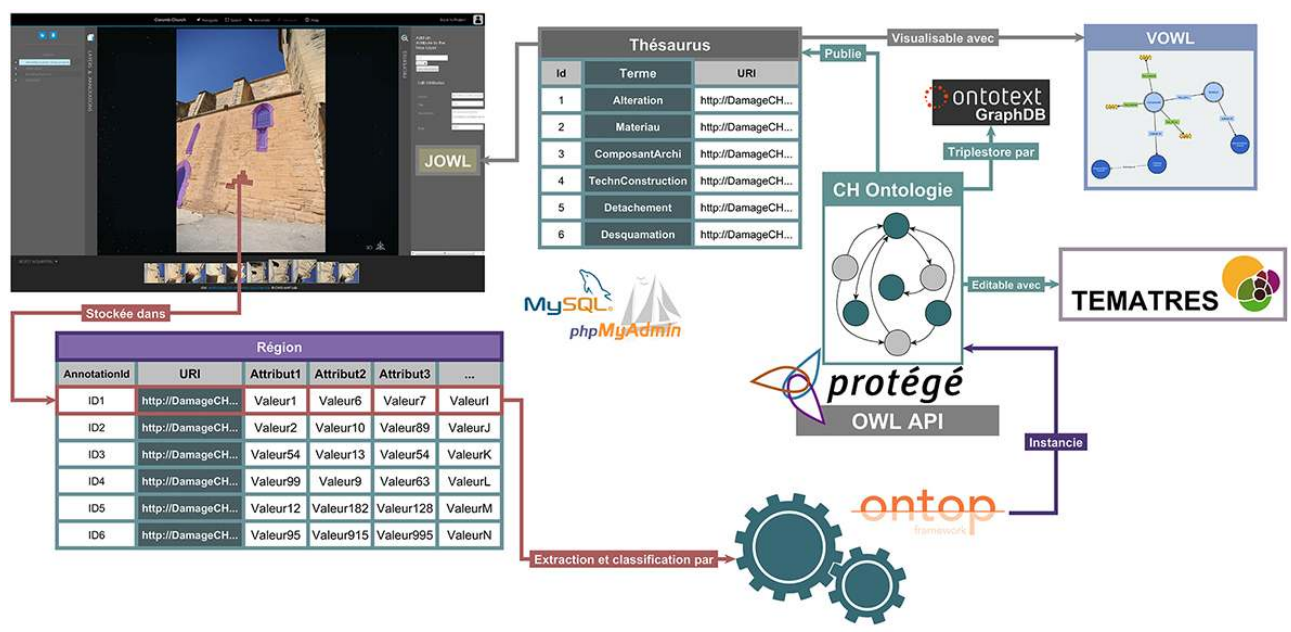

Plan d'implémentation informatique pour le système d'information basé sur une ontologie de domaine.

(c) Tommy Messaoudi, MAP-GAMSAU.

Afin de valider notre approche méthodologique, une expérimentation a été réalisée. Une étape essentielle était de mapper l'ontologie développée sur la base de données. C'est-àdire que grâce à des correspondances SQL/SPARQL de ONTOP, nous pouvons instancier nos classes et donc peupler notre ontologie. Nous avons pu ensuite initier la description de l'état de conservation sur un jeu de données concernant le mur oriental de l'église Saint-Maurice de Caromb. Après le processus de reconstruction 3D, nous avons donc réalisé seize observations scientifiques réparties sur les quatre calques de description 
(fig. 16). Voici les informations que nous pouvons fournir grâce à l'ontologie conçue (fig. 17).

Figure 16
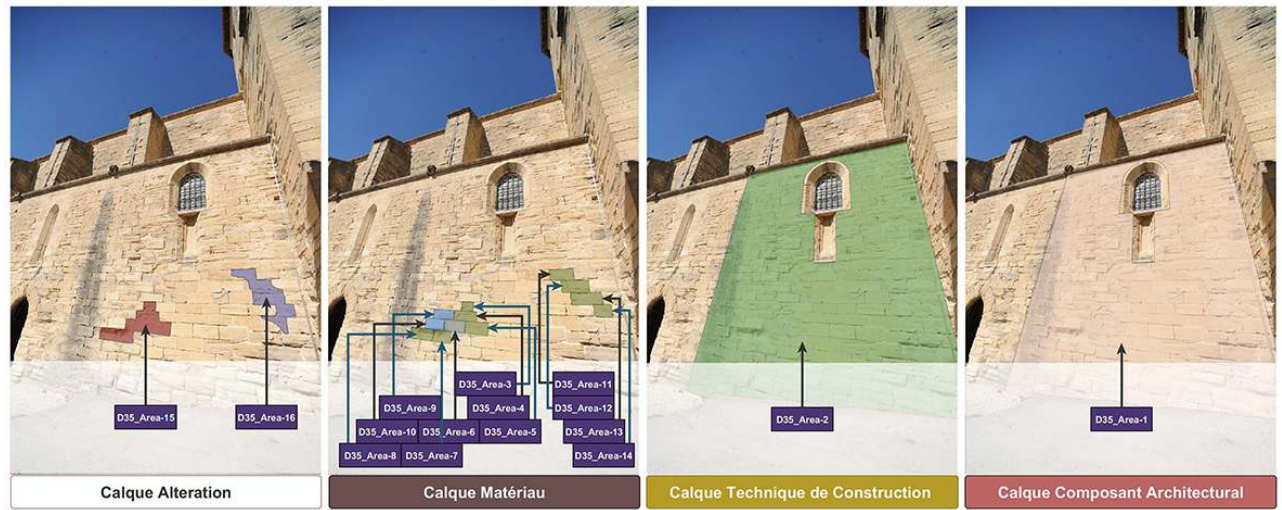

Ensemble des annotations réparties sur les 4 calques de description thématique.

(c) Tommy Messaoudi, MAP-GAMSAU.

La $1^{\text {re }}$ requête (1.) exploite la dimension spatiale : « en prenant en considération l'édifice Église Saint-Maurice, affiche-moi les index en commun des annotations de type Calcaire, et des annotations de type Desquamation en plaque.» Le résultat indique que les régions D35_Area_10 et D35_Area_15 se superposent. Nous pourrons même constater le résultat à travers une visualisation 3D.

Figure 17

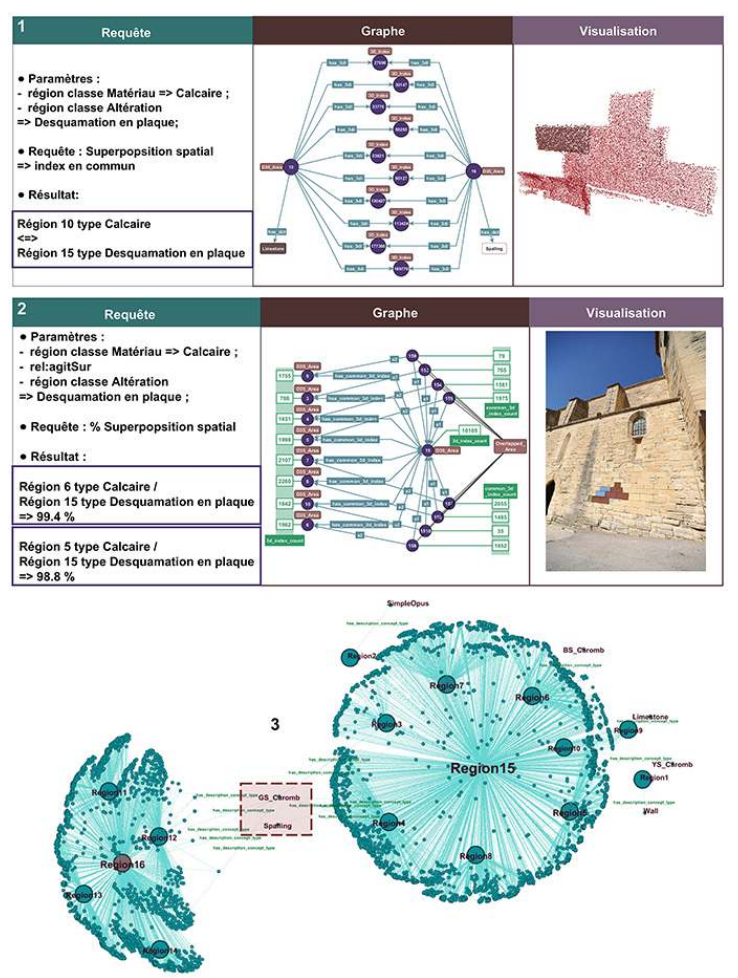

Requêtes (1 et 2) avec leurs résultats et visualisation des résultats sur le logiciel Gephi (3).

(c) Tommy Messaoudi, MAP-GAMSAU. 
La $2^{\mathrm{e}}$ requête (2 .) fournit une information cruciale pour l'expert: "Affiche-moi les annotations de type Calcaire et les annotations de type desquamation en plaque qui se superposent et calcule-moi le degré de recouvrement». Par la combinaison de la superposition spatiale et sémantique, nous pouvons en déduire que la région D35_Area_6 de type «Calcaire» est recouverte à 99,4\% par la région D35_Area_15 de type " Desquamation en plaque » (spalling). Toutes ces requêtes génèrent des triplets qui peuvent être exportés en fichier CSV (Comma-Separated Value). C'est un format de fichier qui sert de donnée d'entrée dans des logiciels de traitement de données graphes. Ce type d'outil tel que Gephi permet d'exploiter l'algorithme de spatialisation Yifan Hu. Il permet de détecter des modalités de relation similaires au sein d'un graphe. Avec les données que nous avons exploitées, nous pouvons répondre à la question: «Sur quel matériau l'altération de type "Desquamation en plaque" agit le plus?». Elle agit davantage sur une pierre calcaire de type «Pierre Grise de Caromb » (GS_Caromb) (3.).

\section{Conclusion et perspectives}

Ce travail a porté sur la proposition d'une ontologie dédiée à l'annotation d'images spatialisées pour le suivi de l'état de conservation du patrimoine bâti. Cette approche, qui repose sur la corrélation de trois dimensions essentielles, a permis de prendre en considération non seulement les aspects quantitatifs mais surtout les aspects qualitatifs à travers les connaissances partagées préétablies. Par cette approche, nous avons pu prendre en compte l'exploitation d'un cadre d'observation scientifique collaboratif pour faire dialoguer les données acquises, les informations extraites et les connaissances formalisées au sein d'un modèle conceptuel innovant. Une vision à long terme serait d'étendre le cadre d'observation collaborative au-delà de la sphère scientifique, vers les sphères des professions du patrimoine (administrateurs, conservateurs, restaurateurs, etc.), voire celle des amateurs du domaine : c'est le crowdsourcing. De plus, au vu de l'essor des technologies mobiles, nous pourrions non seulement proposer à l'expert de réaliser la chaîne de traitement in situ mais également, grâce à la technologie de réalité augmentée, de spatialiser directement des observations sur l'édifice.

Notre contribution, construite au sein d'un contexte spécifique fortement interdisciplinaire, pourra faire émerger, au sein de futures applications, des parcours d'hybridation de méthodes de travail (entre les disciplines). Ils seront essentiels pour l'analyse de plusieurs facteurs déterminants: l'état sanitaire d'un édifice, l'étude des transformations et des évolutions des formes et des phénomènes dans le temps, et la classification des phénomènes observés par le cumul d'observations collectives. Cette ontologie a encore besoin d'être complétée, mais l'intégration innovante des dimensions étudiées permet déjà d'introduire la notion de « continuum informationnel » comme une clé pour l'interconnexion de photographies spatialisées et enrichies sémantiquement, pour instancier une base de connaissances fondée sur le suivi des dégradations du patrimoine architectural. 


\section{NOTES}

1. - DE LUCA, Livio, VALLET, Jean-Marc, BROMBLET, Philippe, et al. « MONUMENTUM : Digital 3D modeling and data management for the conservation of decorated stone buildings ». Dans 13th International Congress on the Deterioration and Conservation of Stone, Paisley, sept. 2016.

2. - BERNERS-LEE, Tim, HENDLER, James, et LASSILA, Ora. "The semantic web». Scientific American, 2001, vol. 284, n 5, p. 34-43. Voir sur le site: https://www-sop.inria.fr/acacia/cours/ essi2006/Scientific\%20American_\%20Feature\%20Article_\%20The\%20Semantic\%20Web_\%20May\% 202001.pdf [consulté le 11/04/2019].

3. - GRUBER, Thomas R. "Toward principles for the design of ontologies used for knowledge sharing ». International journal of human-computer studies, 1995, vol. 43, nº 5-6, p. 907-928.

4. - STEFANI, Chiara, BRUNETAUD, Xavier, JANVIER-BADOSA, Sarah, et al. «3D information system for the digital documentation and the monitoring of stone alteration ». Dans IOANNIDES, Marinos, FRITSCH, Dieter, LEISSNER, Johanna et al. (éd.) Progress in cultural Heritage Preservation. 4th Euro-Mediterranean Conference, Euromed 2012. Berlin : Springer, 2012, p. 330-339.

5. - NIANG, Cheikh, MARINICA, Claudia, LEBOUCHER, Élise, et al. «An ontological model for conservation-restoration of cultural objects ». Dans Digital Heritage, IEEE, 2015, vol. 2, p. 157-160.

6. - CACCIOTTI, Riccardo, BLAŠKO, Miroslav, et VALACH, Jaroslav. «A diagnostic ontological model for damages to historical constructions ». Journal of Cultural Heritage, 2015, vol. 16, $\mathrm{n}^{\circ} 1$, p. 40-48.

7. - HERNANDEZ, Nathalie. Ontologies de domaine pour la modélisation du contexte en recherche d'information. Thèse d'informatique. Toulouse: Institut de recherche en informatique de Toulouse, université Paul-Sabatier, 2005.

8. - DOERR, Martin. "The CIDOC CRM, an ontological approach to schema heterogeneity ». Dans KALFOGLOU, Y., SCHORLEMMER, M., SHETH, A. et al. (éd.). Semantic Interoperability and Integration. Dagstuhl : IBFI, « Dagstuhl Seminar Proceedings », 2005.

9. - BENARD, Amélie. Étude de l'église de Caromb : recherche documentaire et analyse cartographique. Rapport de stage, 2015.

10. - NESPECA, Romina et DE LUCA, Livio. « Point cloud to ontology for software development ». International Archives of the Photogrammetry, Remote Sensing \& Spatial Information Sciences, 2016, vol. 41.

11. - SPARQL Protocol and RDF query language.

12. - aïoli - a 3D semantic annotation platform for the collaborative documentation of cultural heritage objects. A cloud platform developed within the MAP laboratory. Pour plus d'informations, voir le site : www.aioli.cloud [consulté le 11/04/2019].

\section{RÉSUMÉS}

Les pratiques de conservation et restauration de monuments historiques requièrent l'élaboration de diagnostics impliquant une multitude d'experts au sein de contextes d'études 
pluridisciplinaires. L'état de conservation d'un objet patrimonial est décrit au moyen d'observations directes impliquant des sources documentaires et des données analytiques hétérogènes. Parallèlement, si de nouveaux outils permettent aux différents experts de mémoriser et d'analyser leurs observations sur différents supports, les données générées par ces différents acteurs ne sont généralement ni reliées ni spatialisées autour d'un unique support de référence. De plus, aujourd'hui, il est possible de générer des entités 3D denses et précises, mais isolées, ces représentations spatiales ne sont pas suffisantes pour répondre aux exigences d'observation réelle des acteurs de la conservation. Ce constat montre l'absence de cadres opérationnels pour l'extraction d'informations pertinentes pour l'analyse et l'interprétation de l'état de conservation. La nécessité de créer un support d'analyse commun est donc au centre des préoccupations. C'est la raison pour laquelle la création d'une ontologie dans le domaine de la conservation semble être une solution innovante non seulement pour rendre intelligibles des représentations spatiales brutes, mais également pour permettre d'introduire la notion de continuum informationnel. Par l'interconnexion de descripteurs qualitatifs (par une formalisation des connaissances partagées) et quantitatifs, l'ontologie de domaine constitue l'échafaudage conceptuel pour clarifier l'ensemble des interrelations qui permettent par combinaison de décrire les phénomènes de dégradation du bâti.

The conservation and restoration of historic monuments require diagnostic analysis carried out by multidisciplinary teams. The elaboration of this diagnosis of a cultural heritage object involves direct observation, the examination of documentary sources and of diverse types of analytical data. While new tools now allow various experts to store and analyse their observations on different media, the data generated by these different actors is generally not linked to a unique reference, nor spatialized around it. Today, it is possible to generate dense and precise 3D models, but these spatial representations are not adequate for the requirements of real observations of conservation actors. This observation pinpoints the lack of operational frameworks for the capture of information pertaining to the analysis and interpretation of a state of conservation. The need to create a common analytical support is therefore central to these concerns. This is why the creation of an ontology in the field of conservation seems to be an innovative solution not only for making raw spatial representations intelligible, but also for introducing the notion of an informational continuum. By the interconnection of descriptors that are both qualitative (formalising shared knowledge) and quantitative, domain ontology constitutes the conceptual scaffolding which can help clarify all the interrelationships that, by combination, allow for a description of building degradation phenomena.

\section{INDEX}

Keywords : cultural heritage, domain ontology, conservation, semantic annotation, 3D representation, correlation engine, diagnosis

Mots-clés : patrimoine culturel, ontologie de domaine, conservation, annotation sémantique, représentation 3D, continuum informationnel, moteur de corrélation, diagnostic

\section{AUTEURS}

\section{TOMMY MESSAOUDI}

PhD, ingénieur de recherche, UMR CNRS/MCC 3495 Modèles et simulations pour l'Architecture et le Patrimoine messaoudi.tommy@gmail.com 